\title{
Research on the Quality Assurance of Acrylic based on the Optical Coherence Tomography
}

\author{
Zhang Shenjia ${ }^{1,}$, , Liu Guozhong ${ }^{2, b}$ \\ Beijing Information Science and Technology University, Academy of Opto-electronic Engineering \\ and Instrument Science, 100192, Beijing \\ aemail: zhangsj16@163.com, bemail:liuguozhong@bistu.edu.cn
}

Keywords: Acrylic; PMMA; Optical Coherence Tomography; Quality Assurance

\begin{abstract}
According to the problem that the quality assurance of acrylic is not reliable, we use the Michelson interferometer theory to build a Spectral-Domain Optical Coherence Tomography system composed by reference arm, sample arm and spectrometer. Obtaining the 2D and 3D pictures, then measuring the thickness of the acrylic and the width of the nick on surface. Experiments show that the SD-OCT system gets a high resolution and a quick response, providing a method to ensure the quality of the acrylic.
\end{abstract}

\section{Introduction}

Acrylic is also known as Polymeric Methyl Methacrylate(PMMA), having a reputation of "plastic queen" all over the world. Widely used in instrument industry, construction, cleaning tools and many other fields due to its advantages of light weight, high transparency, easy molding, etc. For this reason, accurately detection of acrylic is very important. Quality standard stipulates that the diameter of the defect must within $0.1 \mathrm{~mm}$, allowing 10 defects where diameter between $0.1 \mathrm{~mm}$ and $0.2 \mathrm{~mm}$ [1][2]. Nowadays, the primary detection method is to observe acrylic in a dark box which is slow and not accurate enough to obtain the parameter. SD-OCT system with high resolution and fast response, enable to receive accurate parameters fast [3].

SD-OCT techniques is originated in1990s, based on Michelson interferometer theory [4], interfering by the signal of reference arm and sample arm. Reference arm returns reflected light signal and sample arm returns back scattering or reflected light signal [5]. It is a reliable testing system widely used in biomedical engineering and structure detecting fields.

In this paper, we are researching a more accurate detection method to evaluate acrylic based on SD-OCT. While the resolution of reference arm and sample arm is independent and different [6], we use the SD-OCT system to measure the thickness and the width of acrylic in different way.

\section{Design of the SD-OCT System}

Michelson interferometer formula is applied in SD-OCT system, expressed as:

$$
I(k)=S(k)\left[E_{R}^{2}+2 E_{R} \int_{-\infty}^{+\infty} a(z) \cos (2 k n z) d z+\int_{-\infty}^{+\infty} \int_{-\infty}^{+\infty} a(z) a\left(z^{\prime}\right) \exp \left(i 2 k\left(n z-n z^{\prime}\right)\right) d z d z^{\prime}\right]
$$

In this type: $\mathrm{I}(\mathrm{k})$ is the total interference spectrum; the first term is the interference spectrum of the reference arm itself; the second term is interference spectrum between reference arm and sample arm, which is what we need; the third term is the interference spectrum of the sample arm itself.

In order to obtain 2D and 3D images of acrylic, we need to use the second term of formula (1) and transform it from wave length space to wave number space, then calculating the Fast Fourier Transform, we can find:

$$
H(z)=F(S(k)) \otimes\left(A(z)+A^{*}(-z)\right)
$$

In this type, $\mathrm{H}(\mathrm{z})$ is the FFT of $\mathrm{I}(\mathrm{k})$. Interference signal corresponds to the modulation process, FFT corresponds to demodulation process, then simplified equation (1) and (2), we can find:

$$
I(x)=\int_{-\infty}^{+\infty} I() \cos (2 \pi x) d_{x}
$$




$$
I()=\int_{-\infty}^{+\infty} I(x) \cos (2 \pi x) d_{x}
$$

In this type: $\mathrm{I}(\mathrm{x})$ is the intensity of interference spectrum in the wave length space, $\mathrm{I}(\mathrm{)}$ ) is the intensity in the wave number space, $x$ is optical path difference(opd).

\section{Experimental results}

Measure the thickness of the acrylic. The flow chart expressed as:



Fig.1. Get 2D Image

Acrylic we used is smooth that is to say it has no defects on surface and the thickness is $2000 \mu \mathrm{m}$, which is the most common PMMA material in market. Measuring acrylic with SD-OCT system and collecting interference signal, Figure 2(a) is the interference spectrum when optical path difference is $600 \mu \mathrm{m}$. Figure 2(b) is the Fast Fourier Transform of Figure 2(a), it is clear that opt $=600 \mu \mathrm{m}$. Figure 2(c) is the 2D tomographic picture of the acrylic. In this picture we can see layer 1 corresponds to the surface of the acrylic while layer 2 corresponds to the other side of the acrylic, layer 3 is where the optical path difference equals to 0 , so the thickness of acrylic is the distance between layer 1 and layer 2 while distance layer 1 to layer 3 corresponds to the real optical path difference. Obviously layer 1 is much brighter than layer 2 that is because after layer 1 reflecting the light and also the acrylic absorbs a part of the light, the remaining light is fewer. Figure 2(c) shows us that layer 1 is at 1000, layer 2 is at 930 and layer 3 is at 1021. Every unit refers to $28.5 \mu \mathrm{m}$, then through the calculation we can find out the thickness is $1995 \mu \mathrm{m}$ and the optical path difference is $598.5 \mu \mathrm{m}$. Compared with the theoretical data $2000 \mu \mathrm{m}$ and $600 \mu \mathrm{m}$, the error of the experimental data is just $5 \mu \mathrm{m}$ and $1.5 \mu \mathrm{m}$, it shows the SD-OCT system has high accuracy. 


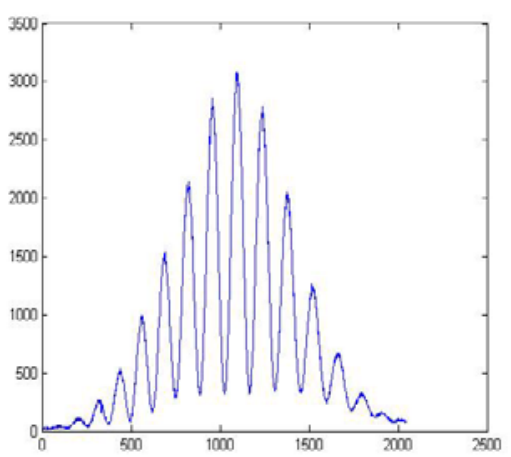

(a) Interference spectrum opt $=600$

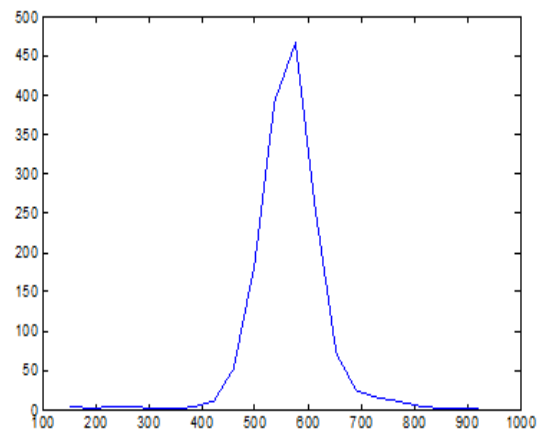

(b) FFT (a)

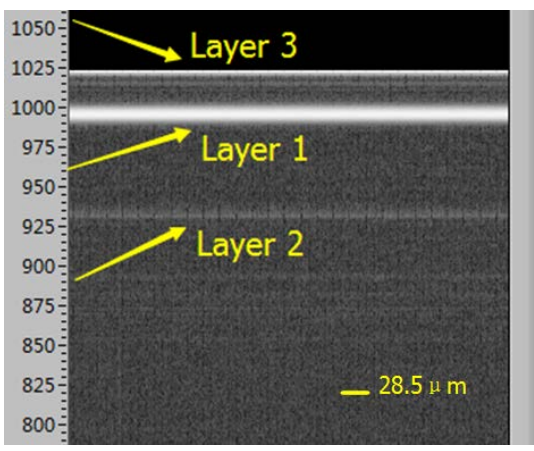

(c) 2D Image to calculate thickness

Fig.2. 2D Image of Acrylic

Measure the width of the nick.

Prepare a Deli No.2041 utility knife, according to the manual we can find the thickness of the blade is 500um, and use this utility knife to make a nick on the surface of the acrylic. After preparing acrylic with a 500um nick, setting a horizontal scanning width 1300um to ensure the nick is within the scanning range of SD-OC. Obtaining the 3D image of the acrylic in Figure 3(c), X refers to the horizontal direction, $\mathrm{Z}$ refers to the depth of the acrylic, XY refers to the one side of the surface. Compared to the layer 1 in Figure 2(b), Figure 3(d) shows a defect performing to a dark line and also not smooth, this defect corresponds to the nick we make. Total scanning range is 1300um, every unit represents 2.6um, so the width of the nick is 499.2um.

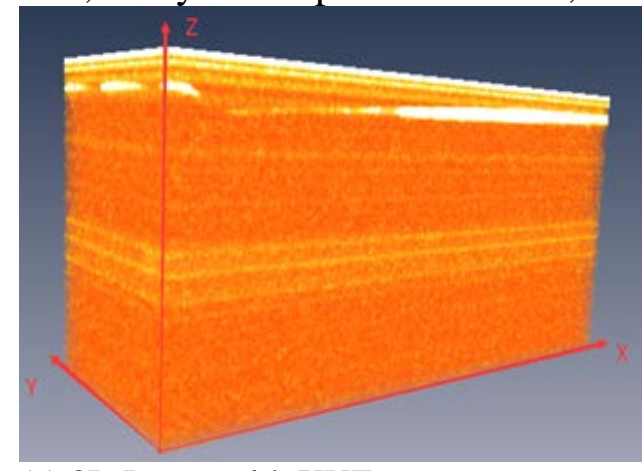

(c) 3D Image with XYZ

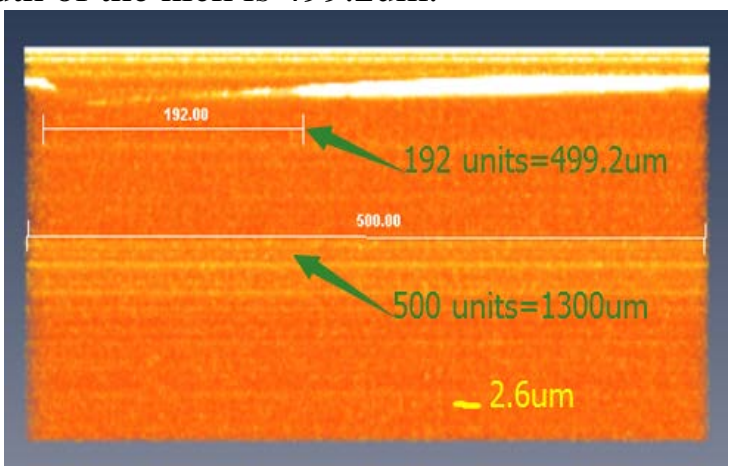

(d) 3D Image to calculate the width of the nick

Fig.3. 3D Image of Acrylic with a nick

\section{Conclusion}

Based on the analysis of the Michelson interferometer theory, designed the Spectral-Domain Optical Coherence Tomography system, reference arm and sample arm return good interference signal, lateral and depth get accurate values. Measuring the thickness and depth of the acrylic, the experimental results show the system has higher accuracy.

\section{Acknowledgement}

In this paper, the research was sponsored by the Nature Science Foundation of China (Project No. 61240057) and subsidized by the Science Research Project of Beijing Educational Committee (KM201311232007).

\section{References}

[1] E.A.Swanson, J.A.lzatt, M.R.Hee, et al.In vivo retinal imaging by optical coherence tomography[J]. Opt.Lett,1993,18:1864-1866

[2] Chashmejahanbin M R, Salimi A, Langroudi A E. The study of the coating adhesion on PP 
surface modified in different plasma/acrylic acid solution[J]. International Journal of Adhesion \& Adhesives, 2014, 49(3):44-50.

[3] Barry Cense, Nader A. Nassif, Johannes F. de Boer, Ultrahigh-resolution high-speed retinal imaging using spectral-domain optical coherence tomography," Opt. Express, 2004, 12: 2435-2447

[4] D. Huang, E. A. Swanson, C. P Lin, et al. Optical coherence tomography. Science.

1991, 254: 1178-1181

[5] Wang, X.J., Milner,T.E., de Boer J.F., et al. Characterization of dentin and enamel by use of optical coherence tomography[J]. Applied Optics.1999,38(10):2092-2096

[6] Lee B H, Min E J, Kim Y H. Fiber-based optical coherence tomography for biomedical imaging, sensing, and precision measurements[J]. Optical Fiber Technology, 2013, 19(6):729-740. 